\section{On the Generalization of True Proportional Navigation}

D. GHOSE

Indian Institute of Science

\begin{abstract}
A simple framework to define generalized true proportional navigation (GTPN) guidance laws is presented. It is shown that this framework subsumes many of the generalizations presented in the earlier literature. The capture regions of a number of GTPN guidance laws are obtained through a rigorous qualitative analysis. The method of analysis is simpler and lends itself directly to an easy geometrical interpretation. A considerable amount of misinterpretation in the previous results, arising out of certain basic misconceptions, are corrected here. It is shown that a logical application of the guidance philosophy, through a minor modification of GTPN to take into account the direction of rotation of the line-of-sight (LOS), contributes substantially to the expansion of the capture region in the relative velocity space. In particular, it is shown that the capture region also extends to the negative closing velocity region, thus making the modified GTPN almost comparable, so far as the domain of capturability of guidance laws is concerned, to the pure proportional navigation (PPN) guidance law. A number of new results on the exact bounds on the capture region are derived and illustrated through examples.
\end{abstract}

Manuscript received February 2, 1993; revised May 6, 1993. IEEE Log No. T-AES/30/2/15496.

Author's Address: Department of Aerospace Engineering, Indian Institute of Science, Bangalore 560 012, India.

$\overline{0018-9251 / 94 / \$ 4.00 ~(C) ~} 1994$ IEEE

\section{INTRODUCTION}

Proportional navigation (PN) has for long been an active area of research for the missile guidance community. A large body of this research concentrates on the linearized version of PN and its variants [1]. However, analysis of the nonlinear PN equations has been the subject of many recent papers. In its nonlinear form, the PN law can be categorized into two major classes: pure proportional navigation (PPN), in which the direction of the commanded missile acceleration is defined with respect to the missile velocity vector; and true proportional navigation (TPN), in which the direction of missile acceleration is defined with respect to the line-of-sight (LOS). Due to its analytical tractability, TPN has generated a large volume of literature. Quite a few of these papers in recent times have concentrated on various generalizations of TPN. The main contributions of the work presented here are four-fold. The first is the formulation of a sufficiently general framework which permits the solution of a large number of meaningful cases of generalized TPN (GTPN) and, in the process, corrects certain misconceptions and errors that have crept into these generalizations in earlier literature. The second contribution is the simplicity of the framework which makes the analysis easier to understand since, in addition to its theoretical rigor, it has an intuitive appeal and lends itself naturally to a straightforward geometrical interpretation. The third contribution of this work is concerned with the fact that in all the earlier literature only a portion of the positive closing velocity region has been considered to be the capture region of GTPN. We show that GTPN also captures from parts of the negative closing velocity region. Further, a minor modification incorporating the direction of LOS rotation expands the capture region even further in the negative closing velocity region, thus making it almost comparable to PPN. Lastly, we obtain exact bounds on the capture region of GTPN with the angle of application of the latax as the variable parameter.

A survey of the literature on TPN reveals that Guelman's [2] was the earliest contribution in this direction, in which an implicit solution of the trajectory parameters was obtained in the closed form and, more importantly, it was shown that when the guidance command is independent of the initial conditions, the capture region remains restricted to a circle in the relative velocity space when TPN is used against a nonmaneuvering target. The importance of the above result lies in the fact that, under reasonable assumptions, PPN can capture a nonmaneuvering target from almost all initial conditions [3, 4]. This fact provided the impetus for further research on TPN in a bid to expand its capture region. This body of research essentially considered a generalized form of TPN and attempted to show that the capture area, under these 
generalizations, expands further. The results in this direction are available in [5-7].

In [2] Guelman considered the simplest form of TPN in which the missile commanded acceleration was applied normal to the LOS, and proportional only to the LOS rate. The resulting capture region was a circle. Other variations to this basic theme occurred when the acceleration was assumed to be also proportional to the initial closing velocity (giving rise to a sector-shaped capture region [8]), and the instantaneous closing velocity (giving rise to a significantly reduced capture region $[8,9])$. In $[6$, 7] a GTPN guidance law was defined which used the angular rate of rotation of a line $\mathrm{L}$ in space, defined with respect to the LOS, in computing the missile acceleration which, in turn, was applied normal to this line. The angular orientation of $L$ with respect to the LOS was assumed to be a function of the instantaneous LOS separation, the instantaneous LOS angle, and perhaps the initial conditions. In [5 and 7] some cases were considered which generalize the original TPN in a straightforward manner. However, in doing so, certain misinterpretations and errors have crept in. For example, in [5] it is claimed that the applied missile acceleration is at a constant angle (defined in [5] as the angular orientation of $L$ with respect to the LOS) from the normal to the LOS. That this is not the case can be easily seen. Further, in [7], the generalized guidance law was reduced to the special case in which the missile lateral acceleration is assumed to be as above. But the solution given is for a guidance law which is different. These points are discussed and clarified later in this work.

Organization is as follows. Section II presents a mathematical formulation of the problem and provides a generalization of the TPN law. In Section III the guidance law is assumed to be independent of initial conditions, and the main capturability theorem for obtaining the capture region of GTPN is stated and proved. In Section IV this result is used to obtain the capture regions for some important generalizations of TPN, and a comparison among them is presented. Exact bounds on the capture region are also obtained here. Conclusions are found in Section V with some discussion on the results and some of their possible extensions.

\section{PROBLEM FORMULATION AND MOTIVATION}

\section{A. Mathematical Model}

The target $T$ and the missile $M$ are assumed to be point mass models on a plane, moving with velocities $V_{T}$ and $V_{M}$, respectively (see Fig. 1). The target is assumed to be a nonmaneuvering one. The missile commanded acceleration is assumed to be applied at an angle $\eta$ from the normal to the LOS. Then the

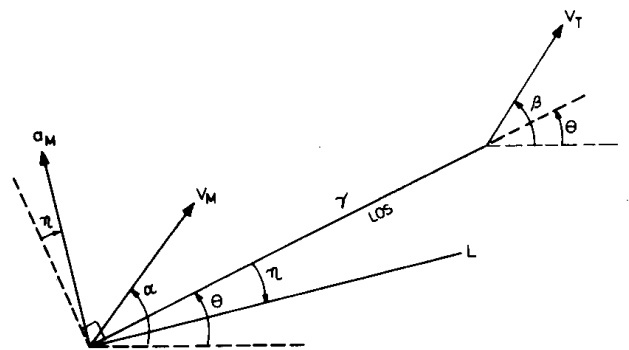

Fig. 1. Missile-target engagement geometry.

equations of motion are obtained as,

$$
\begin{aligned}
V_{r} & =\dot{r}=V_{T} \cos (\beta-\theta)-V_{M} \cos (\alpha-\theta), \\
r(0) & =r_{0}, \quad V_{r}(0)=V_{r 0} \\
V_{\theta} & =r \dot{\theta}=V_{T} \sin (\beta-\theta)-V_{M} \sin (\alpha-\theta), \\
\theta(0) & =\theta_{0}, \quad V_{\theta}(0)=V_{\theta 0} \\
\dot{V}_{M} & =a_{M} \sin (\alpha-\theta+\eta), \quad V_{M}(0)=V_{M 0} \\
\dot{\alpha} & =\left(a_{M} / V_{M}\right) \cos (\alpha-\theta+\eta), \quad \alpha(0)=\alpha_{0}
\end{aligned}
$$

where $V_{r}$ and $V_{\theta}$ are the relative velocities of the target with respect to the missile, along the LOS and normal to the LOS, respectively. According to the original TPN law the missile acceleration is given by

$$
a_{m}=c \dot{\theta}
$$

and is applied in a direction normal to the LOS (i.e., $\eta=0$ in Fig. 1). Most of the results for this case are available in $[2,8,9]$.

\section{B. Generalization of TPN}

Basically, we find that when the applied missile acceleration $a_{M}$ is offset by the angle $\eta \neq 0$ from the normal to the LOS, then $a_{M}$ can be expressed in terms of its components $a_{M r}$ (along the LOS) and $a_{M \theta}$ (normal to the LOS). In this particular case we have $a_{M r}=a_{M} \sin \eta$ and $a_{M \theta}=a_{M} \cos \eta$. Alternatively, one may consider $a_{M r}$ and $a_{M \theta}$ to be independently chosen quantities through which the angle $\eta$ is expressed as $\eta=\tan ^{-1}\left(a_{M r} / a_{M \theta}\right)$. We adopt this alternative for convenience in discussing the ensuing generalizations. Then, analogous to (5), we define

$$
a_{M \theta}=c_{1} \dot{\theta}, \quad a_{M r}=c_{2} \dot{\theta}
$$

with $c_{1}$ and $c_{2}$ being constants which may or may not depend on initial conditions. If $c_{1}$ and $c_{2}$ are independent of initial conditions then we may define a constant $c>0$ as,

$$
c=\left(c_{1}^{2}+c_{2}^{2}\right)^{1 / 2}
$$

with $c_{1}=c \cos \eta$, and $c_{2}=c \sin \eta$. Note that the sign of $c_{1}$ and $c_{2}$ depend on $\eta$. This was the guidance law 
which was described as GTPN in [7, p. 201]. This guidance law had the interpretation that the guidance command was applied normal to a line $L$, which was at a fixed angie $\eta$ from the actual LOS (see Fig. 1), and the commanded acceleration was proportional to the rate of rotation of $\mathrm{L}$ by a constant of proportionality c. However, note that the solution given in [7] for GTPN is not correct. In fact, this solution is actually for the case when $c_{1}$ and $c_{2}$ are dependent on initial conditions and are given by $c_{1}=-N V_{r 0} \cos \psi$ and $c_{2}=$ $N V_{\theta 0} \sin \psi$, where $N>0$ is the navigation constant and $\psi$ is some arbitrary angle. The solution for this guidance law was presented earlier in [5]. It can easily be seen that $\psi \neq \eta$ in general, though in the earlier literature (e.g., [5]) it was implicitly (and erroneously) assumed that $\psi \equiv \eta$. In fact this implicit assumption has led to a considerable amount of misinterpretations of the results and subsequent confusions. To set the record straight, in [5] the angle at which the missile acceleration is applied from the normal to the LOS (denoted here as $\eta$ ) has the following relation with the angle $\psi$,

$$
\tan \eta=\left\{V_{\theta 0} /\left(-V_{r 0}\right)\right\} \tan \psi
$$

which shows that $\eta \neq \psi$ in a general case, contrary to the assumption in [5]. And thus, GTPN in [7] is a different guidance law from the one given in [5].

Here we obtain the complete capture regions for all of the above cases and show the relationships they have with each other. Our method of solution is different from the one given in [5-7] and places more emphasis on rigorous qualitative arguments, rather than on obtaining implicit solutions of trajectory parameters.

\section{GTPN INDEPENDENT OF INITIAL CONDITIONS}

In this section we examine the case in which the missile acceleration according to GTPN is generated independent of the initial conditions, i.e., it is given by (6), with $c_{1}$ and $c_{2}$ being arbitrary constants.

Differentiating (1) and (2) and substituting (3), (4), and (6) appropriately, we obtain the following equations,

$$
\begin{aligned}
& \dot{V}_{r}=\dot{\theta}\left(V_{\theta}-c_{2}\right) \\
& \dot{V}_{\theta}=-\dot{\theta}\left(V_{r}+c_{1}\right) .
\end{aligned}
$$

From (9) and (10), after suitable algebraic manipulations and assuming $V_{\theta} \neq c_{2}$ and $V_{r} \neq-c_{1}$, we obtain

$$
V_{r} \dot{V}_{r}+c_{1} \dot{V}_{r}+V_{\theta} \dot{V}_{\theta}-c_{2} \dot{V}_{\theta}=0 .
$$

Integrating both sides with respect to time,

$$
V_{r}^{2}+2 c_{1} V_{r}+V_{\theta}^{2}-2 c_{2} V_{\theta}=k
$$

with

$$
k=V_{r 0}^{2}+2 c_{1} V_{r 0}+V_{\theta 0}^{2}-2 c_{2} V_{\theta 0}
$$

From (12) and (13) we get,

$$
\left(V_{\theta}-c_{2}\right)^{2}+\left(V_{r}+c_{1}\right)^{2}=\left(V_{\theta 0}-c_{2}\right)^{2}+\left(V_{r 0}+c_{1}\right)^{2} .
$$

This equation shows that the trajectory, from any given initial condition $\left(V_{\theta 0}, V_{r 0}\right)$ in the relative velocity space, remains restricted to the circumference of a circle of radius $p$, given by

$$
p=\left(k+c^{2}\right)^{1 / 2}=\left\{\left(V_{r 0}+c_{1}\right)^{2}+\left(V_{\theta 0}-c_{2}\right)^{2}\right\}^{1 / 2}
$$

and center at $\left(c_{2},-c_{1}\right)$.

Now consider (9) and (10) when $V_{\theta}=0$ and $r \neq 0$. Multiplying $r$ on both sides we obtain,

$$
r \dot{V}_{r}=0, \quad r \dot{V}_{\theta}=0
$$

which implies that $V_{\theta}$ remains at zero and $V_{r}$ remains constant for all future time. Based on this we state the following lemma.

LEMMA 1 If at any finite time the trajectory in the $\left(V_{\theta}, V_{r}\right)$-space reaches a point such that $r \neq 0$, and 1) $V_{\theta}=0$ and $V_{r}<0$, then capture occurs in finite time; 2) $V_{\theta}=0$ and $V_{r} \geq 0$, then capture never occurs.

PRoof. 1) Under this condition the LOS rate is zero the missile is on a collision course, and approaching the target with a constant closing velocity. Hence the engagement ends in a capture in finite time. 2) Under this condition, since the LOS rate is zero, no guidance command is applied. But the missile-target separation is either increasing or remains constant with time. Hence capture does not occur.

Let $\eta \epsilon[0, \pi / 2)$. Consider some possible trajectories in the $\left(V_{\theta 0}, V_{r 0}\right)$-space as shown in Fig. 2. Here, the trajectories are shown for an $\eta>\pi / 4$, and are identified by $\mathcal{C}_{0}, \ldots, \mathcal{C}_{4}$. We may classify all possible trajectories for $c_{1}>0$ and $c_{2}>0$ as follows.

$$
\begin{aligned}
& \mathcal{C}_{0}=\left\{\left(V_{\theta}, V_{r}\right): p=\left(c_{1}^{2}+c_{2}^{2}\right)^{1 / 2}\right\} \\
& \mathcal{C}_{1}=\left\{\left(V_{\theta}, V_{r}\right): p>\left(c_{1}^{2}+c_{2}^{2}\right)^{1 / 2}\right\} \\
& \mathcal{C}_{2}=\left\{\left(V_{\theta}, V_{r}\right): p<\left(c_{1}^{2}+c_{2}^{2}\right)^{1 / 2}, p \geq c_{1}, p \geq c_{2}\right\} \\
& \mathcal{C}_{3}=\left\{\left(V_{\theta}, V_{r}\right): p<\left(c_{1}^{2}+c_{2}^{2}\right)^{1 / 2}, p>c_{1}, p<c_{2}\right\} \\
& \mathcal{C}_{3}^{\prime}=\left\{\left(V_{\theta}, V_{r}\right): p<\left(c_{1}^{2}+c_{2}^{2}\right)^{1 / 2}, p<c_{1}, p>c_{2}\right\} \\
& \mathcal{C}_{4}=\left\{\left(V_{\theta}, V_{r}\right): p<\left(c_{1}^{2}+c_{2}^{2}\right)^{1 / 2}, p<c_{1}, p<c_{2}\right\} .
\end{aligned}
$$

For $\eta \leq \pi / 4$, instead of $\mathcal{C}_{3}$ we have a trajectory of the type $\mathcal{C}_{3}^{\prime}$.

We now determine the direction of movement of a point $\left(V_{\theta}, V_{r}\right)$, with respect to time, on these trajectories. Let $r \neq 0$. Consider the region $V_{\theta}>0$, 
$V_{r} \leq 0$. From (9) and (10) we see that when $V_{\theta}>$ $c_{2}$ and $V_{r}<-c_{1}, \dot{V}_{r}>0$ and $\dot{V}_{\theta}>0$. Similarly, the values of $\dot{V}_{r}$ and $\dot{V}_{\theta}$ for the various regions in the $\left(V_{\theta}, V_{r}\right)$-space is given in Table I. Using these values the direction of movement of a point in the $\left(V_{\theta}, V_{r}\right)$-space is shown in Fig. 2. Applying Lemma 1 , we immediately conclude that the capture region (except the points on the negative $V_{r}$ axis) must lie in the interior of the circle $\mathcal{C}_{0}$. In fact, initial conditions which give rise to trajectories of the nature $\mathcal{C}_{2}$ (i.e., those trajectories which intersect the negative $V_{r}$ axis) definitely lead to capture in finite time. Similarly, using Lemma 1, we conclude that initial conditions lying on the negative $V_{r}$ axis must always lead to capture. Thus, the whole of the negative $V_{r}$ axis belongs to the capture region. Now, consider a point on a trajectory of the type $\mathcal{C}_{1}$. Obviously, according to Lemma 1 , such trajectories never lead to capture. Also, initial conditions lying on the negative $V_{r}$ axis (by virtue of Lemma 1) lead to capture. Consider initial conditions which give rise to trajectories of the type $\mathcal{C}_{4}$. Obviously, these also lead to capture since $V_{r}$ remains negative for all future time and hence capture must occur in time $t_{f}$ with $0<t_{f} \leq r /\left|V_{r \max }\right|$ : where $V_{r \max }$ is the maximum value of $V_{r}$ on the trajectory $\mathcal{C}_{4}$ (shown in Fig. 2). Now, consider initial conditions which give rise to trajectories of the type $\mathcal{C}_{3}$. We see that the value of $V_{r}$ is negative for some portion of the trajectory and positive for the rest. And since $\mathcal{C}_{3}$ does not intersect the $V_{r}$ axis (unlike $\mathcal{C}_{2}$ ) we cannot use Lemma 1 to predict termination or otherwise. Note that, as mentioned earlier, trajectories of the type $\mathcal{C}_{3}$ only occur when $\eta>\pi / 4$ and never for $\eta \leq \pi / 4$. In the rest of the section we prove that trajectories of the type $\mathcal{C}_{3}$ also lead to termination. We also show that both $\mathcal{C}_{3}$ and $\mathcal{C}_{4}$ trajectories have the property that though termination takes place on them, it is not straightforward to identify the point on the trajectory at which this occurs. This is unlike the trajectory $\mathcal{C}_{2}$ where the termination point is the point of intersection of $\mathcal{C}_{2}$ and the negative $V_{r}$ axis. Before we proceed any further note that for $\eta=0$, the above arguments are sufficient to identify the capture region of the original TPN [2] as the interior of $\mathcal{C}_{0}$ with $\eta=0$, i.e., the interior of the circle with radius $c$ and center at $(0,-c)$.

We rewrite (9) and (10) as

$$
\begin{aligned}
\dot{r} & =V_{r} \\
\dot{V}_{r} & =V_{\theta}\left(V_{\theta}-c_{2}\right) / r \\
\dot{V}_{\theta} & =-V_{\theta}\left(V_{r}+c_{1}\right) / r .
\end{aligned}
$$

Now consider Fig. 2 in which the trajectory of $\left(V_{\theta}, V_{r}\right)$ is parameterized by the radius $p$ defined in (15), and an angle $\phi$, as

$$
V_{\theta}=c_{2}+p \cos \phi, \quad V_{r}=-c_{1}+p \sin \phi
$$

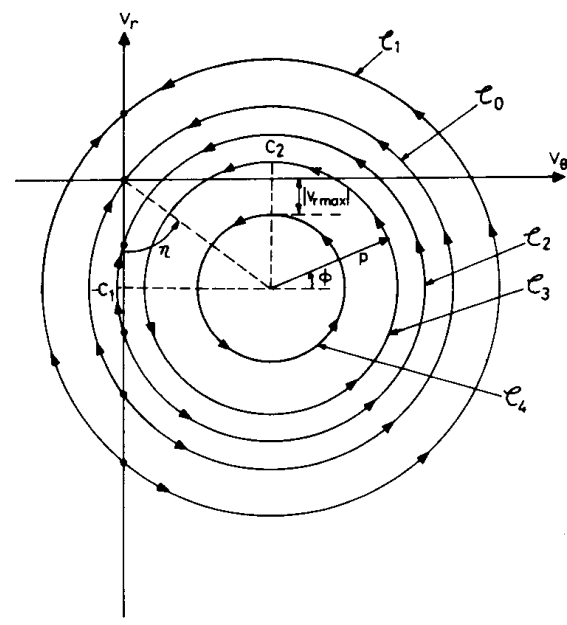

Fig. 2. Trajectories in the $\left(V_{\theta 0}, V_{r 0}\right)$-space.

Differentiating and substituting in (23)-(25) we obtain,

$$
\begin{aligned}
& d r / d t=-c_{1}+p \sin \phi \\
& d \phi / d t=\left(c_{2}+p \cos \phi\right) / r .
\end{aligned}
$$

Eliminating $t$ from (27) and (28) and separating the variables we get,

$$
d r / r=\left\{\left(-c_{1}+p \sin \phi\right) /\left(c_{2}+p \cos \phi\right)\right\} d \phi .
$$

The above equation can now be integrated using standard integration techniques to yield the following results.

Case 1: $\quad p>c_{2}$ (i.e., trajectories of the type $\mathcal{C}_{2}$ ).

$$
\begin{aligned}
\ln \left(\frac{r}{r_{0}}\right)= & \ln \frac{p \cos \phi_{0}+c_{2}}{p \cos \phi+c_{2}}+\frac{c_{1}}{\sqrt{p^{2}-c_{2}^{2}}} \\
& \times \ln \left(\frac{\tan (\phi / 2)-A}{\tan (\phi / 2)+A} \cdot \frac{\tan \left(\phi_{0} / 2\right)+A}{\tan \left(\phi_{0} / 2\right)-A}\right)
\end{aligned}
$$

where

$$
A=\sqrt{\left(p+c_{2}\right) /\left(p-c_{2}\right)} .
$$

Case 2: $\quad p<c_{2}$ (i.e., trajectories of the type $\left.\mathcal{C}_{3} \cup \mathcal{C}_{4}\right)$

$$
\begin{aligned}
\ln \left(\frac{r}{r_{0}}\right)= & \ln \left(\frac{p \cos \phi_{0}+c_{2}}{p \cos \phi+c_{2}}\right)+\frac{2 c_{1}}{\sqrt{c_{2}^{2}-p^{2}}} \\
& {\left[\tan ^{-1}\left\{(1 / B) \tan \left(\phi_{0} / 2\right)\right\}\right.} \\
& \left.-\tan ^{-1}\{(1 / B) \tan (\phi / 2)\}\right]
\end{aligned}
$$

where

$$
B=\sqrt{\left(c_{2}+p\right) /\left(c_{2}-p\right)} .
$$


TABLE I

Direction of Movement of Point in $\left(V_{g q}, V_{r}\right)$-Space

\begin{tabular}{|c|c|c|c|}
\hline \multicolumn{2}{|c|}{ Region } & \multicolumn{2}{|c|}{ Slopes } \\
\hline$v_{\theta}<0$, & $v_{r} \geq 0$ & $\dot{v}_{\theta}>0$, & $\dot{v}_{r}>0$ \\
\hline$v_{\theta}<0$, & $-c_{1}<v_{r}<0$ & $\dot{v}_{\theta}>0$, & $\dot{v}_{r}>0$ \\
\hline$v_{\theta}<0$, & $v_{r}<-c_{1}$ & $\dot{v}_{\theta}<0$, & $\dot{v}_{r}>0$ \\
\hline $0<v_{\theta}: c_{2}$, & $v_{r} \geq 0$ & $\dot{v}_{\theta}<0$, & $\dot{v}_{r}<0$ \\
\hline $0<v_{\theta}<c_{2}$, & $-c_{\uparrow}<v_{r}<0$ & $\dot{v}_{\theta}<0$, & $\dot{v}_{r}<0$ \\
\hline $0<v_{\theta}<c_{2}$, & $v_{r}<-c_{1}$ & $\dot{v}_{\theta}>0$, & $\dot{v}_{r}<0$ \\
\hline$v_{\theta}>c_{2}$, & $v_{r} \geq 0$ & $\dot{v}_{\theta}<0$, & $\dot{v}_{r}>0$ \\
\hline$v_{\theta}>c_{2}$, & $-c_{1}<v_{r}<0$ & $\dot{v}_{\theta}<0$, & $\dot{v}_{r}>0$ \\
\hline$v_{\theta}>c_{2}$. & $v_{r}<-c_{1}$ & $\dot{v}_{\theta}>0$, & $\dot{v}_{r}>0$ \\
\hline$v_{\theta}=0$ & & $\dot{v}_{\theta}=0$, & $\dot{v}_{r}=0$ \\
\hline$v_{\theta}=c_{2}$, & $v_{r}>-c_{1}$ & $\dot{v}_{\theta}<0$, & $\dot{v}_{r}=0$ \\
\hline$v_{\theta}=c_{2}$, & $v_{r}<-c_{1}$ & $\dot{v}_{\theta}>0$, & $\dot{v}_{r}=0$ \\
\hline$v_{\theta}>c_{2}$, & $v_{r}=-c_{1}$ & $v_{\theta}=0$, & $\dot{v}_{r}>0$ \\
\hline$v_{\theta}<c_{2}$. & $v_{r}=-c_{1}$ & $\dot{v}_{\theta}=0$, & $\dot{v}_{r}<0$ \\
\hline$v_{\theta}=c_{2} 0$. & $v_{r}=-c_{1}$ & $\dot{v}_{\theta}=0$, & $\dot{v}_{r}=0$ \\
\hline
\end{tabular}

Note: Roman symbols here correspond to italic symbols in text.

Case 3: $p=c_{2}$.

$$
\begin{aligned}
\ln \left(\frac{r}{r_{0}}\right)= & \ln \frac{1+\cos \phi_{0}}{1+\cos \phi} \\
& +\frac{c_{1}}{c_{2}}\left\{\tan \left(\phi_{0} / 2\right)-\tan (\phi / 2)\right\}
\end{aligned}
$$

Note that the solution in Case 1 holds for all points outside a circle of radius $c_{2}$ and center at $\left(c_{2},-c_{1}\right)$, Case 2 holds for all points interior to this circle, and Case 3 holds for points on this circle. In the following we analyze the equations further to obtain the termination conditions.

Case 1: $p>c_{2}$. 
Equation (30) can be rewritten as

$$
\begin{aligned}
\frac{r}{r_{0}}= & \left(\frac{p \cos \phi_{0}+c_{2}}{p \cos \phi+c_{2}}\right) \\
& \times\left(\frac{\tan (\phi / 2)-A}{\tan (\phi / 2)+A} \cdot \frac{\tan \left(\phi_{0} / 2\right)+A}{\tan \left(\phi_{0} / 2\right)-A}\right)^{\left(c_{1} / \sqrt{p^{2}-c_{2}^{2}}\right)}
\end{aligned}
$$

Obviously, for termination to occur $r$ (and hence, the left-hand side (LHS) of (35)) must become zero. This implies that there must be a finite $t_{f}>0$ for which the right-hand side (RHS) of (35) becomes zero. This can happen if

$$
\tan (\phi / 2)=A=\sqrt{\left(p+c_{2}\right) /\left(p-c_{2}\right)}
$$

which, after simplifications, yields

$$
p \cos \phi=-c_{2}
$$

which is exactly the point at which the trajectory $\mathcal{C}_{2}$ intersects the $V_{r}$ axis (see Fig. 2). However, note that (37) also implies that $p \cos \phi+c_{2}=0$, which appears in the denominator of the RHS of (35). Applying L'Hospital's rule to the following function,

$$
\begin{gathered}
\lim _{\cos \phi \rightarrow\left(-c_{2} / p\right)}\{\tan (\phi / 2)-A\}^{L} /\left(p \cos \phi+c_{2}\right) ; \\
L=c_{1} / \sqrt{p^{2}-c_{2}^{2}}
\end{gathered}
$$

we obtain

$$
\lim _{\cos \phi \rightarrow\left(-c_{2} / p\right)} L\{\tan (\phi / 2)-A\}^{L-1}\left\{\sec ^{2}(\phi / 2) / 2\right\} /(-p \sin \phi) .
$$

The denominator of (39) is not equal to zero since $p>c_{2}$, whereas its numerator becomes zero in the limiting case if and only if (iff) $L>1$, which implies that

$$
p<\sqrt{\left(c_{1}^{2}+c_{2}^{2}\right)}
$$

is the capture condition. It proves that all trajectories of the type $\mathcal{C}_{2}$ lead to a capture. This result matches with the results of the qualitative analysis presented above.

Case 2: $p<c_{2}$.

Equation (32) can be rewritten as

$$
\frac{r}{r_{0}}=\left[\left(\frac{p \cos \phi_{0}+c_{2}}{p \cos \phi+c_{2}}\right) e^{M\left(\phi_{0}\right)}\right] e^{-M(\phi)}
$$

where

$$
M(\phi)=\frac{2 c_{1}}{\sqrt{c_{2}^{2}-p^{2}}} \tan ^{-1}\{(1 / B) \tan (\phi / 2)\} .
$$

Here, $p \cos \phi+c_{2}>0$ and finite for all values of $\phi$, and so the coefficient of $e^{-M(\phi)}$ in the RHS of (41) is always positive. Since at capture $r=0$, we must find the condition under which $e^{-M(\phi)} \rightarrow 0$ as $r \rightarrow 0$.

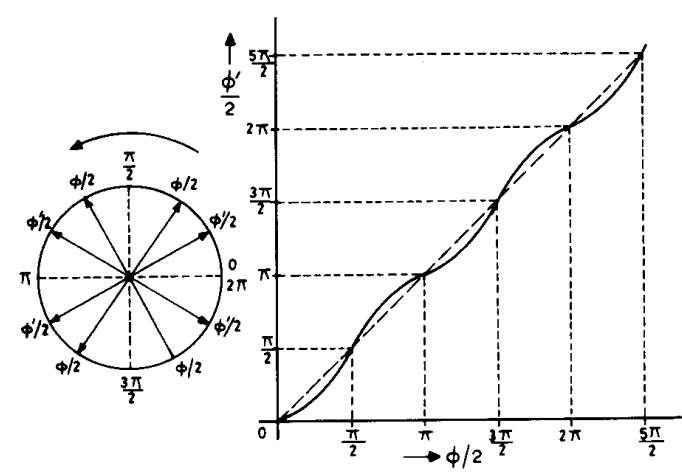

Fig. 3. Variation of $\phi^{\prime}$ with $\phi$.

Obviously, this happens when $M(\phi) \rightarrow \infty$ in finite time. Let us first consider a geometrical interpretation of $M(\phi)$. From (33) we know that $B>1$ and is finite. Define an angle $\phi^{\prime}$ as

$$
\phi^{\prime} / 2=\tan ^{-1}\{(1 / B) \tan (\phi / 2)\} .
$$

Consider what happens to $\phi^{\prime}$ as $\phi$ increases monotonically from $\phi_{0}$ to $\infty$. We note that if the angle $\phi / 2$ defines the angular orientation of a vector in a given quadrant then $\phi^{\prime} / 2$ also defines the angular orientation of another vector in the same quadrant, which either lags or leads the first vector. These angular orientations become equal only when $\phi / 2$ takes values of $n \pi / 2, n=0,1,2, \ldots$. This is shown graphically in Fig. 3 .

Now consider (28) which shows that $d \phi / d t>0$ always (since $p<c_{2}$ ), and hence $\phi$ goes on increasing with time. Suppose it so happens that $\phi \rightarrow \infty$ in finite time. Then from Fig. 3 and the above discussion we know that $\phi^{\prime} \rightarrow \infty$ and hence $M(\phi) \rightarrow \infty$ (provided that $c_{1}>0$ ) thus ensuring termination. To prove that $\phi \rightarrow \infty$ in finite time, we substitute $r$ from (41) into (28) to get

$$
\begin{aligned}
d t / d \phi= & \left\{r_{0}\left(p \cos \phi_{0}+c_{2}\right) e^{M\left(\phi_{0}\right)}\right\} \\
& \times e^{-M(\phi)} /\left(p \cos \phi+c_{2}\right)^{2} .
\end{aligned}
$$

Here, we determine the value of $t$ as $\phi \rightarrow \infty$. From (42) and (43) we obtain,

$$
M(\phi)=c_{1} \phi^{\prime} / \sqrt{c_{2}^{2}-p^{2}} .
$$

From Fig. 3 we know that $\phi^{\prime}$ leads or lags $\phi$ by some angle. The maximum angle of lead or lag is a function of $B$ and is bounded by $\pi$, i.e.,

$$
\sup _{B>1}\left|\phi-\phi^{\prime}\right|=\pi \text {. }
$$

Then,

$$
M(\phi)=c_{1} \phi^{\prime} / \sqrt{c_{2}^{2}-p^{2}}>c_{1}(\phi-\pi) / \sqrt{c_{2}^{2}-p^{2}}
$$


Using these we can write

$$
\begin{aligned}
d t / d \phi= & \left\{r_{0}\left(p \cos \phi_{0}+c_{2}\right) e^{M\left(\phi_{0}\right)}\right\} \\
& \times e^{-M(\phi)} /\left(p \cos \phi+c_{2}\right)^{2} \leq D e^{-E \phi}
\end{aligned}
$$

where,

$$
\begin{aligned}
& D=\left\{r_{0}\left(p \cos \phi_{0}+c_{2}\right) /\left(c_{2}-p\right)^{2}\right\} e^{M\left(\phi_{0}\right)} e^{-E \pi}>0 \\
& E=c_{1} / \sqrt{c_{2}^{2}-p^{2}}
\end{aligned}
$$

From (48) we have

$$
d t \leq D e^{-E \phi} d \phi
$$

Integrating both sides and substituting suitable initial conditions, we obtain

$$
t \leq(D / E)\left(e^{-E \phi_{0}}-e^{-E \phi}\right) .
$$

As $\phi \rightarrow \infty$, the RHS reaches a finite value, i.e.,

$$
\lim _{\phi \rightarrow \infty} t \leq(D / E)\left(e^{-E \phi_{0}}\right) .
$$

Since the RHS is finite, it implies that $\phi \rightarrow \infty$ within a finite time and hence the termination occurs. Thus, all trajectories of the type $\mathcal{C}_{3} \cup \mathcal{C}_{4}$ terminates in finite time with a capture of the target if $c_{1}>0$.

Case 3: $p=c_{2}$.

Equation (34) can be rewritten as

$$
r / r_{0}=\left\{\left(1+\cos \phi_{0}\right) /(1+\cos \phi)\right\} e^{N\left(\phi_{0}\right)} e^{-N(\phi)}
$$

where,

$$
N(\phi)=\left(c_{1} / c_{2}\right) \tan (\phi / 2) .
$$

Applying L'Hospital's rule we observe that $r \rightarrow 0$ as $\phi \rightarrow \pi$. This implies that capture occurs when the trajectory touches the negative $V_{r}$ axis.

To summarize, the above analysis showed that, apart from the engagement geometries in which the initial condition lies on the negative $V_{r}$ axis, capture occurs only for $c_{1}>0$. The actual analysis (Cases 1-3) were done for $c_{1}>0$ and $c_{2}>0$. However, identical results determining the capture conditions for $c_{1}>0$ and $c_{2}<0$ can be obtained in an exactly similar manner. Combining all the above results, we state the main capturability theorem of this paper.

THEOREM 1 A missile pursuing a nonmaneuvering target with a generalized TPN guidance law given by (6), captures the target if 1) $V_{r 0}<0$ and $V_{\theta 0}=0$; or 2) $c_{1}>0$, and the initial condition lies in the interior of a circle of radius $\left(c_{1}^{2}+c_{2}^{2}\right)^{1 / 2}$ and center at $\left(c_{2},-c_{1}\right)$ defined in the $\left(V_{\theta 0}, V_{r 0}\right)$-space, i.e., it lies in the interior of the circle given by the equation,

$$
\left(V_{\theta 0}-c_{2}\right)^{2}+\left(V_{r 0}+c_{1}\right)^{2}<c_{1}^{2}+c_{2}^{2}
$$

PROOF. Follows directly from the analysis given above.

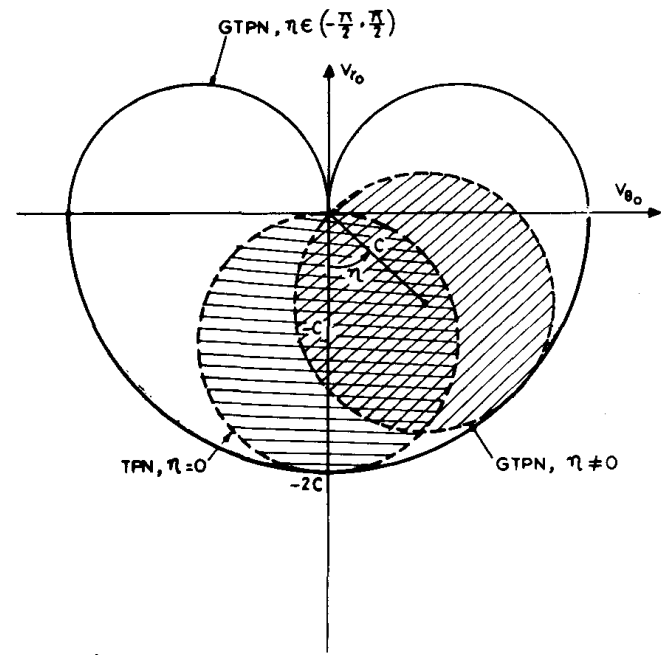

Fig. 4. Capture region of GTPN independent of initial conditions.

The above theorem gives rise to a number of interesting and new results. The foremost is that unlike the original TPN [1] guidance law (in which $c_{1}>0$ and $c_{2}=0$ ), the GTPN can also ensure capture from some initial conditions at which the closing velocity is negative. This was a point which had been overlooked in the previous literature. Further, it should be noted that this theorem subsumes the results given in [5 and 7] on GTPN and permits the analysis of other generalizations too. In order to obtain an easy and natural comparison of these generalizations with the original TPN, let us define the constant $c>0$ as in (7) so that

$$
c_{1}=c \cos \eta, \quad c_{2}=c \sin \eta
$$

with $\eta \epsilon(-\pi / 2, \pi / 2)$ which ensures that $c_{1}>0$ is always satisfied. This was the case mentioned in [7, p. 201] without the correct solution. The correct solution shows that the capture region is a circle of radius $c$ and center at $(c \sin \eta,-c \cos \eta)$ in the $\left(V_{\theta}, V_{r}\right)$-space. We observe that, for a fixed $\eta$, the area of the capture region remains exactly the same as in the original TPN. However, for $\eta \neq 0$, some points with negative closing velocity are also included in the capture region while some points, which were inside the capture region for $\eta=0$, are now excluded from the capture region. If $\eta$ is not fixed and is a freely chosen parameter in the guidance law then the complete capture region is the union of all capture regions for each $\eta \epsilon[-\pi / 2, \pi / 2]$. This is shown in Fig. 4.

\section{GTPN DEPENDENT ON INITIAL CONDITIONS}

The main result in the previous section (as given in Theorem 1) is sufficient to obtain capture regions for a number of special cases. First let us consider the case given at the end of the previous section (i.e., the 


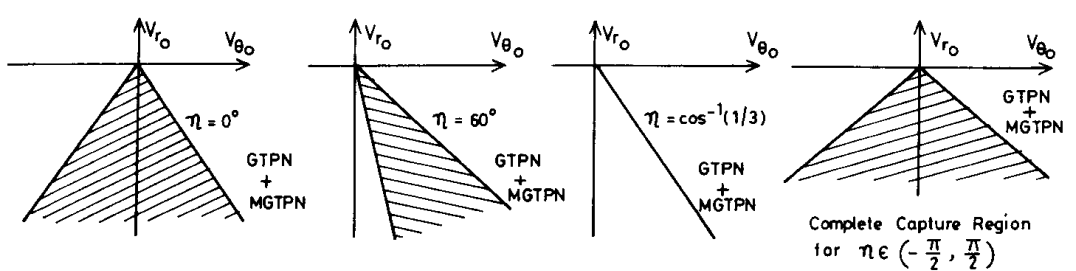

(a)

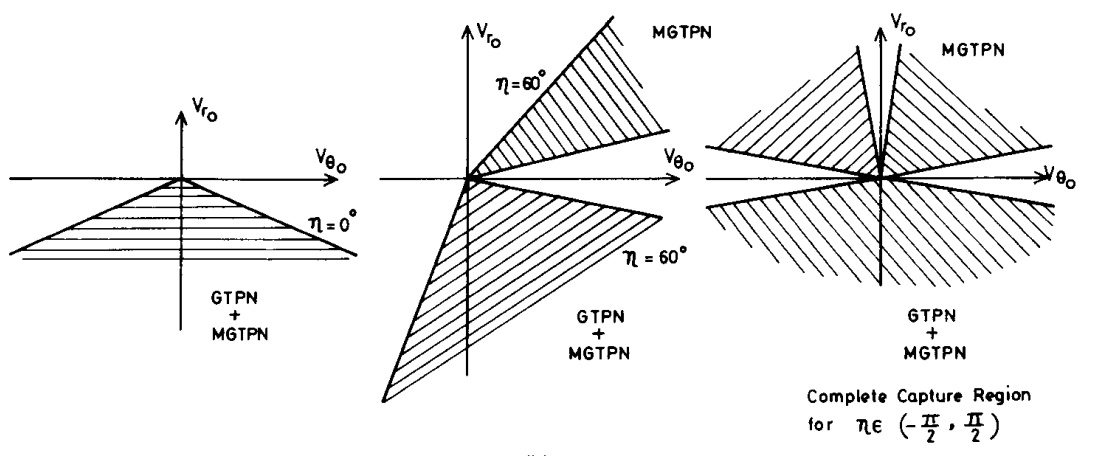

(b)

Fig. 5. Capture region of GTPN and MGTPN dependent only on $V_{r 0}$. (a) $N=0.75$. (b) $N=3$.

one in $[7$, p. 201]). We assume that the constant of proportionality $c$ in the guidance law is a function of the initial closing velocity (as in the case of the original TPN and also in [7]), i.e.,

$$
c=-N V_{r 0}
$$

where, $N$ is the navigation constant. Then, from (56), we have

$$
c_{1}=-N V_{r 0} \cos \eta, \quad c_{2}=-N V_{r 0} \sin \eta
$$

Note that by virtue of Theorem 1 , capture occurs only when $V_{r 0}<0$ (since this ensures $c_{1}>0$ ) for this particular guidance law. Note also that the whole of the negative $V_{r}$ axis is always included in the capture region no matter what the values of $N$ and $\eta$ are. In the subsequent analysis we assume this implicitly and by "capture region" we refer to the collection of capture points apart from the ones on the negative $V_{r}$ axis. Substituting (58) in the capture circle equation (55) in Theorem 1 we get,

$$
\left(V_{\theta 0}+N V_{r 0} \sin \eta\right)^{2}+\left(V_{r 0}-N V_{r 0} \cos \eta\right)^{2}<N^{2} V_{r 0}^{2}
$$

which can be simplified to yield

$$
V_{\theta 0}^{2}+V_{r 0}^{2}(1-2 N \cos \eta)+2 N V_{\theta 0} V_{r 0} \sin \eta<0 .
$$

Application of certain basic results in analytic geometry $[10]$ leads us to the following results about the capture region boundary. 1) If $N<1 /(1+\cos \eta)$ then the capture region does not exist. 2) If $N \geq$ $1 /(1+\cos \eta)$ then the capture region exists and is a sector-shaped region whose boundaries are given by

$$
\begin{array}{r}
\left(-V_{r 0}\right)[N \sin \eta-\sqrt{\{N(1-\cos \eta)+1\}\{N(1+\cos \eta)-1\}}]<V_{\theta 0} \\
\quad<\left(-V_{r 0}\right)[N \sin \eta+\sqrt{\{N(1-\cos \eta)+1\}\{N(1+\cos \eta)-1\}]} .
\end{array}
$$

In Figs. 5(a) and 5(b) a number of capture region boundaries for various values of $\eta \epsilon[0, \pi / 2)$ are shown for $N=0.75$ and $N=3$, in the negative $V_{r 0}$ region. It is obvious that when $\eta \epsilon(-\pi / 2,0]$ the capture region is a mirror image (about the $V_{r}$ axis) of the capture region for the corresponding positive value of $\eta$. As $\eta$ increases from 0 to $\pi / 2$ the capture region becomes asymmetric about the $V_{r}$ axis and finally migrates into the right side of the $V_{r}$ axis. For $N \leq 1$, when $\eta=\cos ^{-1}\{(1-N) / N\}$ the capture region reduces to a single line. This we can see in Fig. 5(a). If the parameter $\eta$ is not fixed and can be freely chosen then, as in the previous section, the actual capture region is the union of all the capture regions for various $\eta$ and its boundary can be found by maximizing the RHS of (61) with respect to $\eta$. This yields the result that the maximum boundary on the right side is obtained when

$$
\eta=\cos ^{-1}(1 / 2 N) \text {. }
$$

This has been used to obtain the envelope of the complete capture region in Figs. 5(a) and 5(b) for $\eta \epsilon(-\pi / 2, \pi / 2)$. These figures show that the capture area exists only in the region of negative $V_{r 0}$, though in the previous section (Figs. 2 and 4) there was a considerable capture area in the positive $V_{r 0}$ region too. The reason for this apparent anomaly lies in the way in which $c_{1}$ is defined in (58). In Section III, 
$c_{1}>0$ was assumed regardless of the initial condition $V_{r 0}$, but in (58) $c_{1}<0$ when $V_{r 0}$ is positive and thus there is no capture region in the positive $V_{r}$ region. Therefore, the correct way to define the guidance law is to define $c$ as,

$c=N\left|V_{r 0}\right| ; \quad c_{1}=N\left|V_{r 0}\right| \cos \eta, \quad c_{2}=N\left|V_{r 0}\right| \sin \eta$

We call this the MGTPN (modified GTPN). For the original TPN $(\eta=0)$ this problem did not arise as the whole of the capture region was in the negative $V_{r 0}$ region. Note that, with this modification, the capture region in the negative $V_{r 0}$ region for MGTPN remains the same as obtained above. But, in addition, we also get some capture area in the positive $V_{r 0}$ region. Substituting (63) in the capture equation (55) we obtain,

$$
\left(V_{\theta 0}-N\left|V_{r 0}\right| \sin \eta\right)^{2}+\left(V_{r 0}+N\left|V_{r 0}\right| \cos \eta\right)^{2}<N\left|V_{r 0}\right|^{2}
$$

which can be simplified further for $V_{r 0}>0$ to obtain,

$$
V_{\theta 0}^{2}+V_{r 0}^{2}(1+2 N \cos \eta)-2 N V_{\theta 0} V_{r 0} \sin \eta<0
$$

as the capture region. An identical analysis leads us to the capture area in the positive $V_{r 0}$ region.

1) If $N<1 /(1-\cos \eta)$, then the capture region does not exist. 2) If $N \geq 1 /(1-\cos \eta)$, then the capture region exists and is the interior of a sector-shaped region lying on one side of the $V_{r}$ axis, and is given by,

$$
\begin{aligned}
V_{r 0}[N & \sin \eta-\sqrt{\{N(1-\cos \eta)-1\}\{N(1+\cos \eta)+1\}}]<V_{\theta 0} \\
& <V_{r 0}[N \sin \eta+\sqrt{\{N(1-\cos \eta)-1\}\{N(1+\cos \eta)+1\}}]
\end{aligned}
$$

This capture area is also shown in Fig. 5(b) in the positive $V_{r 0}$ region, for $N=3$ and $\eta=60^{\circ}$. For $N=$ 0.75 , the capture region for $V_{r 0}>0$ does not exist. Note that the capture region of MGTPN is the same as the capture region of GTPN in the negative $V_{r 0}$ region. Here we find that when $\eta$ is not fixed, the envelope of the capture region for $\eta \epsilon[0, \pi / 2)$ is obtained by putting $\eta=\pi / 2$ in the RHS of (66). This is shown in Fig. 5(b) as the envelope of the complete capture region in the positive $V_{r 0}$ region for $\eta \epsilon(-\pi / 2, \pi / 2)$. Note that there is a sector-shaped region symmetric about the $V_{r 0}$ axis which has to be excluded.

The above analysis obtains the complete capture region for GTPN and MGTPN guidance laws from the main result stated in Theorem 1 . In the following analysis we show that the complete capture region for the GTPN as given in [5] can also be obtained from Theorem 1. For this we define (exactly as in [5]),

$$
c_{1}=-N V_{r 0} \cos \psi, \quad c_{2}=N V_{\theta 0} \sin \psi,
$$

where $\psi$ is an arbitrary angle between 0 and $\pi / 2$. Here too, capture can occur only when $V_{r 0}<0$. The actual

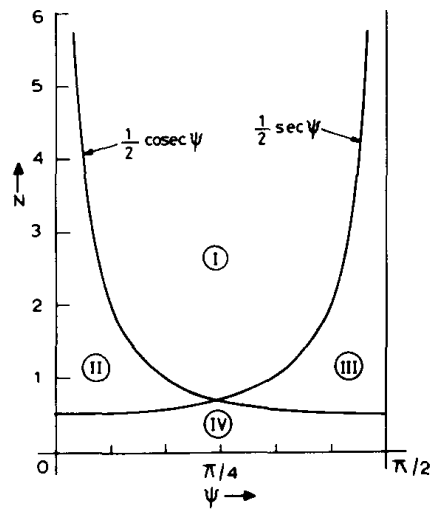

Fig. 6. Capture conditions in the $(\psi, N)$-space for GTPN dependent on both $V_{\theta 0}$ and $V_{r 0}$.

angle, at which the missile acceleration is directed, is given by $\eta$ in (8). Now, substituting in (55) we obtain,

$$
\begin{gathered}
V_{\theta 0}^{2}(1-N \sin \psi)^{2}+V_{r 0}^{2}(1-N \cos \psi)^{2} \\
<N^{2}\left(v_{r 0}^{2} \cos ^{2} \psi+V_{\theta 0}^{2} \sin ^{2} \psi\right)
\end{gathered}
$$

which can be simplified to yield

$$
V_{\theta 0}^{2}(1-2 N \sin \psi)+V_{r 0}^{2}(1-2 N \cos \psi)<0
$$

which, in turn, immediately leads to the capture condition given in [5, eqn. (14)], which however is not sufficient to provide all the information on the actual shape of the capture region in the $\left(V_{\theta 0}, V_{r 0}\right)$-space. In the following analysis, we obtain the capture region by determining the combinations of $N$ and $\psi$, with $N>0$, $\psi \epsilon[0, \pi / 2]$, for which the capture region exists. We have the following cases.

Case I: $\{N>0.5 \sec \psi, N>0.5 \operatorname{cosec} \psi\}$. The whole of the $V_{r 0}<0$ region is the capture region.

Case II: $\{N>0.5 \sec \psi, N<0.5 \operatorname{cosec} \psi\}$. The symmetric sector-shaped region defined by,

$$
\begin{aligned}
\left|V_{\theta 0}\right| & <\sqrt{(2 N \cos \psi-1) /(1-2 N \sin \psi)}\left(-V_{r 0}\right), \\
V_{r 0} & <0
\end{aligned}
$$

is the capture region.

Case III: $\{N<0.5 \sec \psi, N>0.5 \operatorname{cosec} \psi\}$. The region defined by,

$$
\begin{aligned}
\left|V_{\theta 0}\right| & >\sqrt{(1-2 N \cos \psi) /(2 N \sin \psi-1)}\left(-V_{r 0}\right), \\
V_{r 0} & <0
\end{aligned}
$$

is the capture region.

Case IV: $\quad\{N \leq 0.5 \sec \psi, N \leq 0.5 \operatorname{cosec} \psi\}$. The capture region does not exist.

In Fig. 6, these different cases of capture (or noncapture) are shown in terms of $N$ and $\psi$. In 

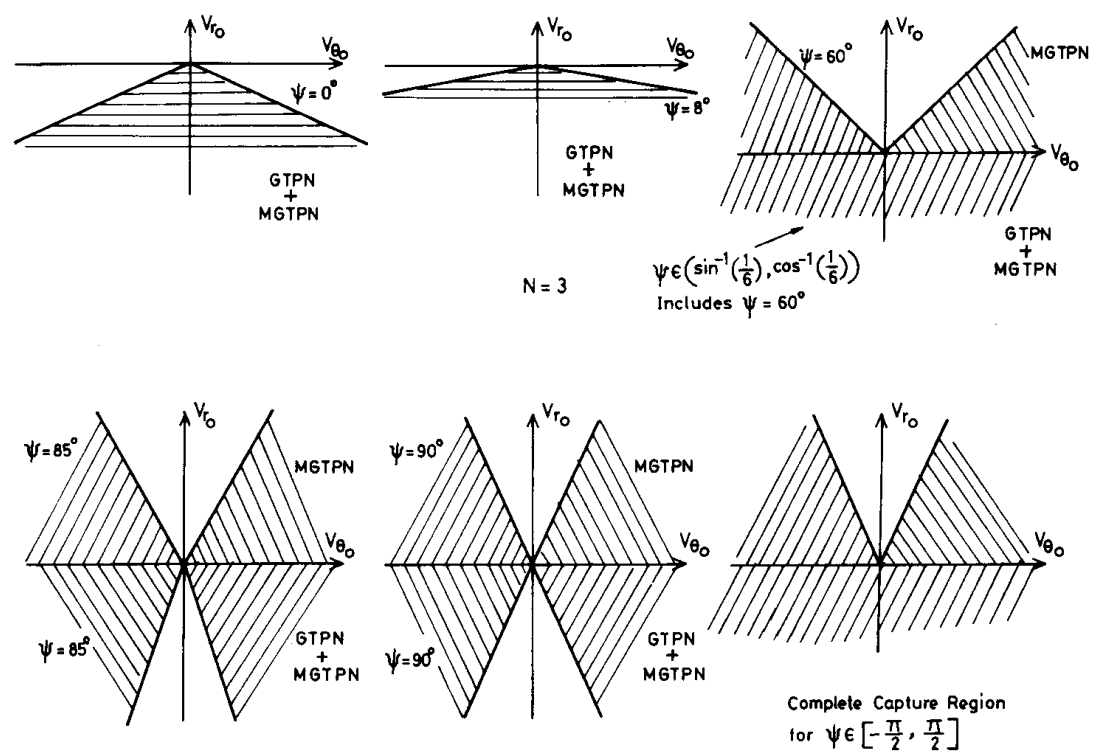

Fig. 7. Capture region of GTPN and MGTPN dependent on both $V_{\theta 0}$ and $V_{r 0}$.

Fig. 7 we show some capture regions for $N=3$ and various values of $\psi$. We note that in this case if $0<$ $\psi<\sin ^{-1}(1 / 2 N)$, the capture region is sector shaped $\left(\eta=8^{\circ}\right.$ is shown in the figure), when $\sin ^{-1}(1 / 2 N) \leq$ $\psi \leq \cos ^{-1}(1 / 2 N)$ then the capture region is the whole of the negative $V_{r}$ region whereas when $\psi>$ $\cos ^{-1}(1 / 2 N)$ the capture region is the negative $V_{r}$ region, excluding a sector-shaped area symmetric about the $V_{r}$ axis.

As in the earlier analysis, here also we observe that the guidance law implemented in this fashion does not have any capture area in the positive $V_{r 0}$ region. We make a similar modification to define,

$$
c_{1}=N\left|V_{r 0}\right| \cos \psi
$$

with $c_{2}$ as defined in (67). Now, substituting in (55), we get,

$$
\begin{gathered}
V_{\theta 0}^{2}(1-N \sin \psi)^{2}+\left(V_{r 0}+N\left|V_{r 0}\right| \cos \psi\right)^{2} \\
<N^{2}\left(\left|V_{r 0}\right|^{2} \cos ^{2} \psi+V_{\theta 0}^{2} \sin ^{2} \psi\right) .
\end{gathered}
$$

Obviously, this will give rise to the same capture region as before in the region $V_{r 0}<0$. In the following, we examine the possibility of capture in the region $V_{r 0}>0$. Then (73) can be simplified to yield,

$$
V_{\theta 0}^{2}(1-2 N \sin \psi)+V_{r 0}^{2}(1+2 N \cos \psi)<0 .
$$

Since $(1+2 N \cos \psi)>0$ for all $\psi \epsilon[0, \pi / 2]$ the capture region can be obtained according to the following. 1) If $N>0.5 \operatorname{cosec} \psi$, then the capture region is given by,

$$
\begin{aligned}
|V \theta 0| & >\sqrt{(2 N \cos \psi+1) /(2 N \sin \psi-1)} V_{r 0}, \\
V_{r 0} & \geq 0 .
\end{aligned}
$$

2) If $N<0.5 \operatorname{cosec} \psi$, the capture region does not exist.

This capture region for $N=3$ and $\psi=60^{\circ}$ and $85^{\circ}$ is shown in Fig. 7. The whole of the positive $V_{r 0}$ region excluding a sector-shaped region symmetric about the $V_{r 0}$ axis is the actual capture region. The complete capture region is the largest capture region, obtained by substituting $\psi=\pi / 2$ in (75) and is also shown in Fig. 7.

Note that for $\psi \epsilon[-\pi / 2,0]$, the capture area in the region $V_{r 0}<0$ is a subset of the capture area for $\psi=0$, and in the region $V_{r 0}>0$, it does not exist.

From the above analyses and illustrative examples we can easily see that the two generalizations discussed above are not the same and they produce different capture regions in the $\left(V_{\theta 0}, V_{r 0}\right)$ space. Moreover, the logical modification of GTPN adopted above expands the capture region considerably in the negative closing velocity region.

\section{CONCLUDING REMARKS}

In this paper we propose a method for obtaining the capture region of GTPN guidance laws. The main result of this analysis is shown to be applicable in determining the capture regions of a number of generalizations of TPN available in the literature, and in providing an easy comparison among them. Some prevalent misconceptions and errors in the previous literature have also been corrected here. It is found that regardless of the generalization adopted, the capture region for GTPN still remains a proper subset of that for the PPN guidance law. This is well in accord with the analysis given earlier in [11]. 
However, in this paper we show that after a minor but logical modification of GTPN it becomes almost comparable to the PPN law in so far as the capture region is concerned. Another outcome of this study is that it presents the capture region as a natural and robust basis for performance comparison among various guidance laws. Recently this has been used for performance comparison in the case of maneuvering targets too $[12,13]$. Some possible extensions of this kind of research may involve some of the variants and extensions of PN, such as the augmented PN [14] and the modern guidance scheme [15], which have been designed explicitly for maneuvering targets. Their analysis on the same basis, in a nonlinear framework, is expected to yield interesting results.

\section{REFERENCES}

[1] Zarchan, P. (1990)

Tactical and strategic missile guidance.

In Progress in Aeronautics and Astronautics, Vol. 124 Washington, DC: AIAA Inc., 1990.

[2] Guelman, M. (1976)

The closed-form solution of true proportional navigation. IEEE Transactions on Aerospace and Electronic Systems, AES-12, 4 (July 1976), 472-482.

[3] Guelman, M. (1971)

A qualitative study of proportional navigation.

IEEE Transactions on Aerospace and Electronic Systems, AES-7, 4 (July 1971), 637-643.

[4] Becker, K. (1990)

Closed-form solution of pure proportional navigation. IEEE Transactions on Aerospace and Electronic Systems, 26, 3 (May 1990), 526-533.

[5] Yang, C.-D., Yeh, F.-B., and Chen, J.-H. (1987)

The closed-form solution of generalized proportional navigation.

Journal of Guidance, Control, and Dynamics, 10, 2

(Mar--Apr. 1987), 216-218.
[6] Yang, C.-D., and Yeh, F.-B. (1987)

Closed-form solution for a class of guidance laws. Journal of Guidance, Control, and Dynamics, 10, 4 (July-Aug. 1987), 412-415.

[7] Yang, C.-D., Hsiao, F.-B., and Yeh, F.-B. (1989) Generalized guidance law for homing missiles. IEEE Transactions on Aeraspace and Electronic Systems, 25, 2 (Mar. 1989), 197-212.

[8] Dhar, A., and Ghose, D. (1993) Capture region for a realistic TPN guidance law. IEEE Transactions on Aerospace and Electronic Systems, 29, 3 (July 1993), 995-1003.

[9] Yuan, P.J., and Chern, J.-S. (1992)

Solutions of true proportional navigation for maneuvering and nonmaneuvering targets.

Journal of Guidance, Control, and Dynamics, 15, 1 (Jan.-Feb. 1992), 268-271.

[10] Krasnov, M., Kiselev, A., Makarenko, G., and Shikin, E. (1990)

Mathematical Analysis for Engineers, Vol. 1.

Moscow: Mir Publishers, 1990.

[11] Shukla, U. S., and Mahapatra, P. R. (1990)

The proportional navigation dilemma-pure or true? IEEE Transactions on Aeraspace and Electronic Systems, 26, 2 (Mar. 1990), 382-392.

[12] Ghose, D.

True proportional navigation with maneuvering target. IEEE Transactions on Aeraspace and Electronic Systems, 30, 1 (Jan. 1994), 229-237.

[13] Ghose, D. (1994)

Analysis of capturability of the TPN class of guidance laws.

Technical Report, AE/GI/001/94 Department of Aerospace Engineering, Indian Institute of Science, Bangalore, India, (Jan. 1994).

[14] Siouris, G. M. (1974)

Comparison between proportional and augmented proportional navigation.

Nachrichtentechnische Zeitschrift, 27, 7 (July 1974), 278-280.

[15] Cottrell, R. G. (1972)

Optimal intercept guidance for short-range tactical missile. ALAA Journal, 9, 7 (July 1971), 1414-1415.

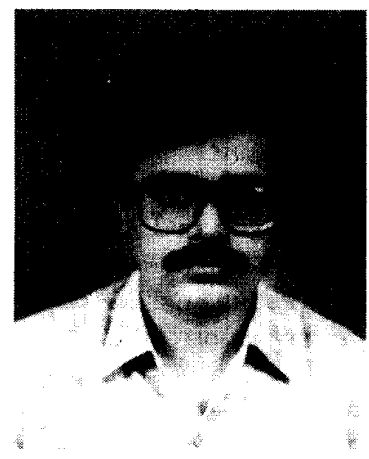

Debasish Ghose received the B.Sc.(Engg) in electrical engineering from the Regional Engineering College, Rourkela, India, in 1982, and the M.E. and Ph.D. degrees, also in electrical engineering, from the Indian Institute of Science, Bangalore, India, in 1984 and 1990, respectively.

From 1984 to 1987 he worked as a Scientific Officer in the Joint Advanced Technology Programme at the Indian Institute of Science, where he is presently an Assistant Professor in the Department of Aerospace Engineering. His research interests are in the areas of guidance and control, dynamic game theory, and distributed computing. 